\title{
Distribution of Silymarin in the Fruit of Silybum marianum L.
}

Duma Hlangothia ${ }^{1}$, Fawzia Abdel-Rahman ${ }^{2}$, Thao Nguyen ${ }^{1}$, Kevin Anthony ${ }^{1}$ and Mahmoud A Saleh ${ }^{1}$

${ }^{1}$ Department of Chemistry, Texas Southern University, 3100 Cleburne Street, Houston, Texas 77004, USA

${ }^{2}$ Department of Biology, Texas Southern University, 3100 Cleburne Street, Houston, Texas 77004, USA

"Corresponding author: Saleh MA, Department of Chemistry, Texas Southern University, 3100 Cleburne street, Houston, Texas, 77004, USA, Tel: 713 3131912; Fax: 713 3137824; E-mail: saleh_ma@tsu.edu

Received date: October 24, 2016; Accepted date: November 11, 2016; Published date: November 14, 2016

Copyright: (c) 2016 Hlangothia D, et al. This is an open-access article distributed under the terms of the Creative Commons Attribution License, which permits unrestricted use, distribution, and reproduction in any medium, provided the original author and source are credited.

\begin{abstract}
The fruit of Silybum marianum or milk thistle is known for its rich contents of flavonlignan compounds known as silymarin. Silymarin has been recognized for centuries as "liver tonics" and is well known to prevent or reverse hepatotoxicity. Silymarin is usually extracted from the defatted fruits in methanol in a yield of less than $2 \%$. Fluorescent microscopy, scanning electron microscopy, accurate mass spectrometry as well as infra-red and Raman spectroscopy were used for mapping silymarin in the fruit. Silymarin was found to be only located in the pericarp section of the fruit. Extraction of silymarin from the pericarp gave higher yields of more than $6 \%$ and did not require defatting.
\end{abstract}

Keywords: Antioxidants; Liver protection; Flavonlignan; Milk thistle; Raman spectral mapping; SEM

\section{Introduction}

Silybum marianum L. Gaertn (Asteraceae) is an annual to biennial herb originates from mountains of the Mediterranean and North African regions where it forms scrub on a rocky base [1]. Currently it grows wild throughout Europe, North Africa, the Americas, and Australia [2,3]. It has several common names such as milk thistle, blessed milk thistle, Marian thistle, Mary thistle, Saint Mary's thistle, Mediterranean milk thistle, variegated thistle and Scotch thistle. The flower heads of the plant are mostly red Purple (some cultivars have white flower) the flower head-bud is about $4 \mathrm{~cm}$ long and $12 \mathrm{~cm}$ wide. $S$. marianum plants flower from June to August in the North or December to February in the Southern Hemisphere. $S$. marianum fruits vary from white to nearly black. The plant is cultivated on a large scale in Austria, Germany, Hungary, Poland, China and Argentina for the production of raw material for the pharmaceutical industry. The fruits of $S$. marianum are one of the most important herbal liver medicines. The plant is the most studied medicinal plant in the treatment of liver disease. Fruits of $S$. marianum have been used for more than 2000 years to treat a range of liver and gallbladder disorders, including hepatitis, cirrhosis, and jaundice, and to protect the liver against poisoning from chemical and environmental toxins, including snake bites, insect stings and mushroom poisoning. Extracts of $S$. marianum fruits have been recognized for centuries as "liver tonics" and are well known to prevent or reverse hepatotoxicity of reactive drug metabolites or naturally occurring toxins [4]. The plant is sometimes also used as a decorative element in gardens, and its dried flower heads may be used for the decoration of dry bouquets [5]. The fruits are harvested in July-August after blooming. The medicinal part of the plant is the fruits often referred to as "seeds". Silymarin is the major bioactive constituent of the fruit extract of Silybum marianum, consisting of a mixture of one flavanonol (taxifolin) and six flavonolignans (silychristin, silydianin, silybin A, silybin B, isosilybin A and isosilybin B) [6-9], however, a very recent investigation by Hohmann group revealed the presence of up to 23 flavolignan components [10]. The fruit is also known to be rich in lipids with up to $25 \%$ weight/weight [11] which interfere with the extraction of silymarin and has to be removed from the fruits (defatting) prior to silymarin extraction using nonpolar organic solvents. Little is known about the location of silymarin in the fruit of Silybum marianum, but most of them relayed on using microscopic histology that were lacking structural information or spectral data [12], or were based on mechanical separation of fruit parts and extracting them with appropriate solvent which still did not show exactly the precise location of silymarin in the fruit [13-14].

The objective of this study was to use microscopic spectroscopy for mapping the distribution of silymarin in the fruit of Silybum marianum for the purpose of improved efficiency of extracting silymarin.

\section{Experimental Section}

\section{Fruits of Silybum marianum}

Certified Silybum marianum fruit were purchased from Frontier Natural Products CO-OP (2012 Frontier, Norway, IA 52318 USA) patch \# 912.3128. Seeds were washed with deionized water, ultrasonicated, and air dried prior to investigation.

\section{Stereomicroscopy}

Whole fruits were first observed using stereomicroscope for morphology, shape, size, and color parameters. Free hand longitudinal sections were obtained from the whole fruits and observed using Nikon's SMZ25 Fluorescence Stereomicroscope with EGFP Bandpass filter having excitation at $450-490 \mathrm{~nm}$ and emission at $500-550 \mathrm{~nm}$ (Nikon Instruments Inc., Melville, NY, USA) equipped with NIS Elements High Content Analysis Microscope Imaging Software.

\section{Scanning Electron Microscopy (SEM)}

Sections of dried fruits were hand-made using a razor blade, mounted on aluminum stubs using double-sided adhesive carbon tape 
and were sputtered with a thin layer $(20 \AA)$ of gold using Desk V HP Danton Vacuum system (Danton Vacuum LLC, Moorestown, NJ, USA) under argon gas. Coated fruit sections were examined and imaged by the scanning electron microscopy Evex Global Nano SEM at an accelerated voltage of $20 \mathrm{KV}$.

\section{Infrared Spectroscopy}

FT Raman and FT infrared spectra were acquired using Nicolet IS50 (Thermo Scientific). IR was acquired using IS50 ATR for solid samples.

\section{High Performance Thin Layer Chromatography (HPTLC)}

Methanolic extracts of the fruit shell and the embryo cotyledons surrounded with the endosperm layer were examined by HPTLC along with standard silymarin solution on silica gel plates in $10 \mathrm{~mm}$ bands at $40 \mu \mathrm{g} / \mathrm{band}$ (HPTLC Silica Gel 60F254 $20 \times 10$ Merck K GaA 6427 Darmstadt, Germany). Plate was developed with chloroform, acetone and formic acid 75:16.5:8.5 v/v, dried and observed at a UV of 254 and $366 \mathrm{~nm}$. Plates were also derivatives in $1 \%$ vanillin reagent, $(1 \mathrm{~g}$ of Vanillin, $100 \mathrm{~mL}$ of ethanol mixed with $5 \mathrm{~mL}$ of conc. Sulphuric acid and $95 \mathrm{~mL}$ of ethanol), and the plate was dried in the oven at $100^{\circ} \mathrm{C}$ for $5 \mathrm{~min}$, after which spots were visualized under white light and $366 \mathrm{~nm}$ wavelengths. All applications including spotting, development, derivatization and imaging were performed using the Camag automated instruments (Automatic TLC Sampler 4, Rep Pro Star 3 and ADC 2 Automatic Developer) and win Cats-Planar Chromatography manager software (CAMAG USA, Denver, CO).

\section{Accurate Mass Spectrometry}

Accurate Mass were measured using LC-QTOF Agilent 6530 system equipped with 1290 binary pump, Phenomenex Kinetex C18 100 mm $\times 3.0 \mathrm{~mm} 2.6 \mu \mathrm{m}$ column. Solvents A was made of methanol with $0.1 \%$ formic acid; B was $0.1 \%$ formic acid in water run in a gradient protocol starting at $30 \%$ A to $60 \%$ A over 15 min with a flow $0.2 \mathrm{~mL} / \mathrm{min}$. Ion source ESI+Agilent Jet Stream Technology in positive ionization mode. The Jet Stream ESI source was operated in positive/negative mode, and instrument parameters were set as follows: sheath gas temperature, $350^{\circ} \mathrm{C}$; sheath gas flow, $8 \mathrm{~L} / \mathrm{min}$; nebulizer, $20 \mathrm{psi}$; dry gas temperature, $300^{\circ} \mathrm{C}$; dry gas flow, $5 \mathrm{~L} / \mathrm{min}$; and capillary entrance Voltage, $3500 \mathrm{~V}$. Fragmentor and Skimmer were operated at 190 and $65 \mathrm{~V}$, respectively. The MS scan data were collected at a rate of 1.02 spectra/s in the range of $\mathrm{m} / \mathrm{z}$ 100-2000. All the MS data were collected with Mass Hunter Data Acquisition B.06.00 (Agilent Technologies), and Mass Hunter Qualitative Analysis B.06.00 (Agilent Technologies) was applied to identify silymarin components. All EICs were obtained with $\pm 10 \mathrm{ppm}$ $\mathrm{m} / \mathrm{z}$ expansion.

\section{Results and Discussion}

Fruits of Silybum marianum are small elongated ovate and slightly compressed dorsoventrally. Fruits vary in color from light pale yellow to brown and dark reddish brown with smooth shiny surface, the exocarp (pericarp epidermis) consisting of elongated polygonal cells. Average dimensions of the fruits are $6.8 \mathrm{~mm}$ in length and $3.0 \mathrm{~mm}$ in width with an average weight of $2.8 \mathrm{~g} / 100$ fruit (Figure 1).

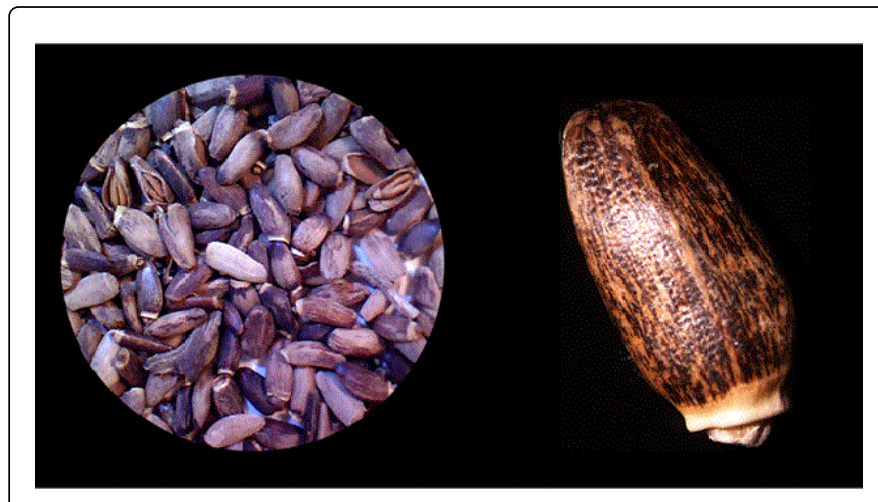

Figure 1: Fruits of Silybum marianum.

Scanning Electron Microscopy (SEM) examination of the fruit sections revealed that $S$. marianum consist of the pericarp, testa epidermis, endosperm and the embryo. The pericarp is subdivided into exocarp, mesocarp and endocarp. Exocarp is the outermost layer (pericarp epidermis), mesocarp which makes the pericarp parenchyma, and these cells contain dark tannins responsible for the spotted appearance of the fruits. The sclerenchyma cells of the endocarp contain large calcium oxalate prismatic crystals [9]. Testa epidermis layer consisted of elongated macrosclereids and followed by the subepidermal tissue. The endosperm forms a single layer of cells that surrounds the embryo's two cotyledons with many elongated cells filled with fat droplets (Figure 2).

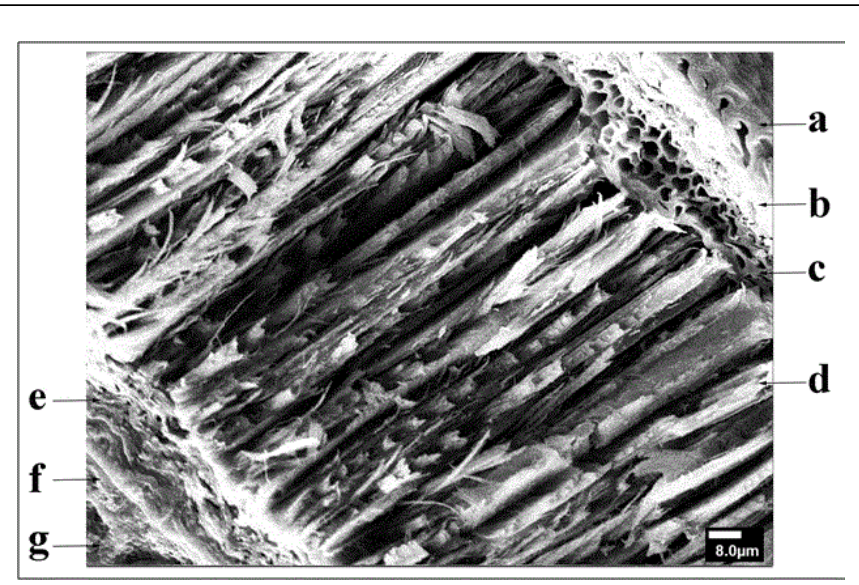

Figure 2: SEM photomicrograph of Silybum marianum fruit cross section: a) exocarp; b) mesocarp; c) endocarp; d) testa epidermis; e) subepidermal layer; f) endosperm; g) embryo.

Examination of the fruit sections using fluorescent stereomicroscope using Nikon's SMZ25 Fluorescence Stereomicroscope revealed that silymarin is located in the pericarp layer of the fruit. Certified silymarin standard was found to give a strong green fluorescent at 500-550 nm when exited at $450-490 \mathrm{~nm}$ using the EGFP Bandpass filter; fats and proteins did not show any emission using that filter. Fluorescent mapping of the fruit longitudinal section is shown in Figure 3. Fluorescence emission under the stereomicroscope produced a diffused image which is difficult to locate the exact locations of the silymarin as appeared as continues green 
Page 3 of 4

band in the pericarp, the apical region of the fruit, and to lesser extent at the testa epidermal layer.
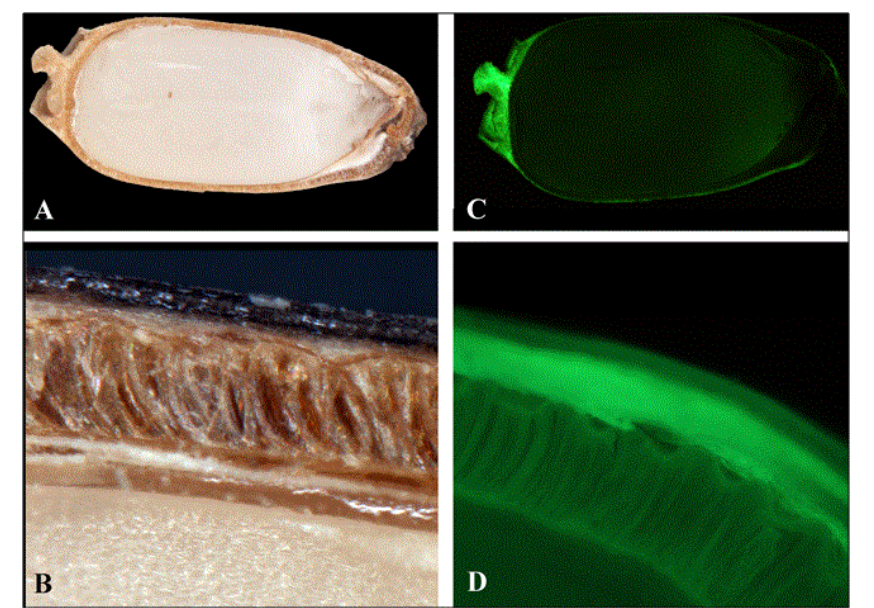

Figure 3: Stereomicroscope micrographs of Silybum marianum longitudinal sections: $\mathrm{A}$ and $\mathrm{B}$ no fluorescent applied; C and D epifluorescence showing the distribution of silymarin in the pericarp.

Raman spectra mapping showed precisely that silymarin is only located in the layer of the pericarp (endocarp) that included the crystals, and also in the apical region. It appears as none continues band that reflects the distribution of the crystals within the endocarp tissues. The red color indicated the highest concentrations of the silymarin and the blue color indicates the lowest as shown in Figure 4.

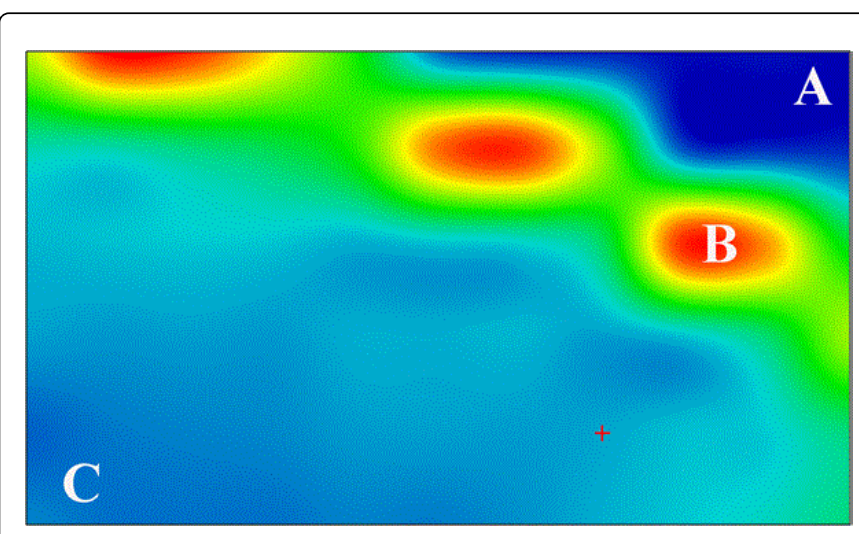

Figure 4: Raman spectra mapping of silymarin in $S$. marianum section: A) outside the fruit surface; B) pericarp; C) embryo.
To confirm our results, the fruits were mechanically separated as shell layer and endosperm/embryo layers and each layer was extracted with methanol to examine their contents of silymarin. The two extracts were examined first by HPTLC and revealed that the endosperm/ embryo layer did not have any silymarin and was mostly consisted of proteins and lipids. On the other hand, the shell layer was very rich in silymarin as shown in Figure 5. Yield of silymarin in the shell was $6 \%$ compared to $2 \%$ in the whole fruits.

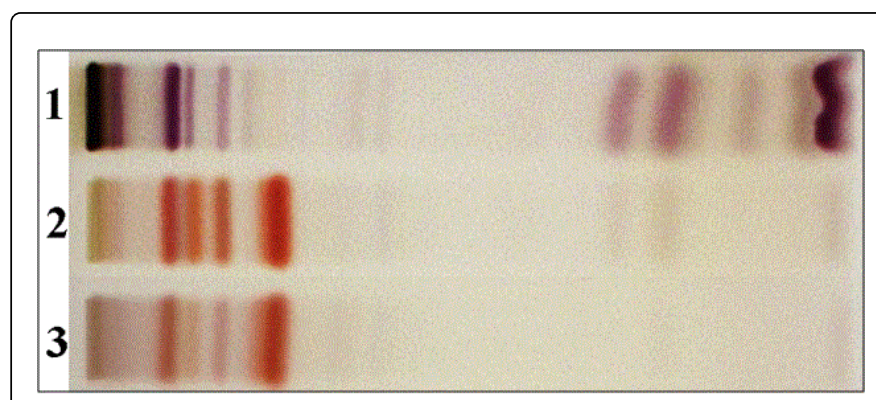

Figure 5: HPTLC of Silybum marianum extracts: 1) endosperm/ embryo; 2) fruit shell; 3) standard silymarin.

FTIR of the solid materials of the shell and the other inside layers of the fruits (endosperm and embryo) showed that IR spectra confirmed that silymarin is located only in the shell of the fruits. The chromone carbonyl group $\left(1660 \mathrm{~cm}^{-1}\right)$, the aromatic ether linkage group (1160 $\left.\mathrm{cm}^{-1}\right)$, the phenolic hydroxyl group $\left(3500 \mathrm{~cm}^{-1}\right)$, the keto group (1745 $\left.\mathrm{cm}^{-1}\right)$ and the lactone ring group $\left(1752 \mathrm{~cm}^{-1}\right)$ characteristic absorption for silymarin were only detected in the shell of the fruits. Raman bands found in the shell of the fruits but not in the endosperm layers were the symmetric aromatic ring stretching at $1605 \mathrm{~cm}^{-1}$, the $\mathrm{C}=\mathrm{C}$ ring stretching at $1649 \mathrm{~cm}^{-1}$, and the asymmetric ring vibration at 1500 $\mathrm{cm}^{-1}$

Further confirmation was carried out by examining the methanol extracts of the shell and the inner layers of the fruits using accurate mass QTOF analysis showing again that silymarin components exist only in the shell as presented in Table 1 .

\begin{tabular}{|c|c|c|c|c|c|c|c|}
\hline Accurate Mass & Taxifolin & Silychristin & Silydianin & Silybin A & Silybin B & Isosilybin A & Isosilybin B \\
\hline \multicolumn{8}{|l|}{ Positive lons } \\
\hline$\{M+1\}+$ & 305.0649 & 483.1278 & 483.1287 & 483.1275 & 483.1287 & 483.1275 & 483.1282 \\
\hline \multicolumn{8}{|l|}{ Negative lons } \\
\hline$\{M-1\}-$ & 303.0513 & 481.1129 & 481.1137 & 481.1141 & 481.1137 & 481.1137 & 481.1134 \\
\hline
\end{tabular}

Table 1: Accurate mass data of silymarin individual compounds. 
Citation: Mahmoud Saleh (2016) Distribution of Silymarin in the Fruit of Silybum marianum L. Pharm Anal Acta 7: 511. doi: $10.4172 / 2153-2435.1000511$

Page 4 of 4

\section{Conclusion}

Micro spectroscopy using fluorescence microscopy and Raman microscopy of the fruit sections showed that silymarin is located exclusively in the pericarp layer or the fruit shell. Extraction of silymarin from the mechanical separated fruit sections into its inner part (endosperm and embryo) and the seed shell (pericarp) with methanol using microwave assisted extraction revealed that silymarin is mostly located in the pericarp with a recovered yield of $6 \%$. No silymarin was recovered from the endosperm/embryo layer. The obtained results suggested that using the pericarp is more efficient in extracting silymarin than using the whole fruit. It also reduces consumption of solvents necessary for defatting of the fruit while the endosperm/embryo part, which is rich in fatty oil, can be used for production of biodiesel fuel.

\section{Acknowledgments}

This publication was made possible, in part, by research infrastructure support from grant number 2G12MD007605 from the NIMHD/NIH.

\section{References}

1. Boulos L (2000) Flora of Egypt. Al Hadara Publishing Inc, Cairo, Egypt, pp: 125-182.

2. Everist SL (1974) Poisonous Plants of Australia Revised edition. Angus \& Robertson, Sydney, Australia.

3. Parsons WT, Cuthbertson EG (2001) Noxious Weeds of Australia (2nd edn.) Angus \& Robertson. Sydney, Australia.
4. Svoma E (1998) Studies on the embryology and gynoecium structures in Drimys winteri (Winteraceae) and some Annonaceae. Plant Syst Evol 21: 205-229.

5. Quave CL (2012) Medicinal Plant Monographs. Emory University Press, USA.

6. Anthony Kl, Saleh MA (2013) Free Radical Scavenging and Antioxidant Activities of Silymarin Components. Antioxidants 2: 398-407.

7. Anthony K, Subramanya G, Uprichard S, Hammouda F, Saleh MA (2013) Antioxidant and anti-hepatitis $\mathrm{c}$ viral activities of commercial milk thistle food supplements. Antioxidants 2: 23-36.

8. Anthony K, Saleh MA (2012) Chemical profiling and antioxidant activity of commercial milk thistle food supplements. J Chem Pharm Res 4: $4440-4450$.

9. Upton R, Graff A, Jolliffe G, Länger R, Williamson E (2010) American Herbal Pharmacopoeia: Botanical Pharmacognosy Microscopic Characterization of Botanical Medicines. Milk Thistle Fruits, CRC Press, 616-618.

10. Csupora D, Csorbaa A, Hohmanna J (2016) Recent advances in the analysis of flavonolignans of Silybum marianum. J Pharm Biomed Anal 130: 301-317.

11. Harrabi S, Romdhane H, Daassa M, Fellah H (2015) Fatty acid and triacylglycerol compositions of milk thistle seeds growing wild in Tunisia (Silybum marianum L.). Acta Aliment Hung 44: 304-310.

12. Cappelletti EM, Caniato R (1984) Silymarin localization in the fruit and seed of Silybum marianum L. Gaertn. Herba Hungarica 23: 53-66.

13. Stoiljković Z, Petrović S, Ilić B (2007) Examination of localization of silymarin and fatty oil in Silybum marianum (L.) Gaertn fruit. Chem Ind Chem Eng 13: 55-59.

14. AbouZid SF, Chen SN, McAlpine JB, Friesen B, Pauli GF (2016) Silybum marianum pericarp yields enhanced silymarin products. Fitoterapia 112: 136-143. 